\title{
COกE).(OES
}

CIÊNCIA E TECNOLOGIA

\section{HOTELARIA HOSPITALAR: UM ESTUDO DE CASO SOBRE O SETOR DE ALIMENTOS E BEBIDAS EM UM HOSPITAL CEARENSE}

\author{
Victor Hugo Santos de CASTRO ${ }^{1}$, Thieli Mota Rodrigues ${ }^{1}$ \\ ${ }^{1}$ Instituto Federal de Educação, Ciência e Tecnologia do Ceará - IFCE \\ <vsantosdecastro@yahoo.com.br><thielim@hotmail.com>
}

DOI: $10.21439 /$ conexoes.v15i0.1254

\begin{abstract}
Resumo. Esta pesquisa aborda o setor de alimentos e bebidas no âmbito da hotelaria hospitalar. O objetivo geral do estudo foi analisar a organização do setor de alimentos e bebidas de um hospital público cearense. Os objetivos específicos foram: averiguar quais os principais alimentos oferecidos pela instituição e sua relação com a reabilitação e hospitalidade; identificar como ocorre a elaboração do cardápio e os profissionais envolvidos neste processo; verificar a procedência e armazenamento dos alimentos. Quanto à metodologia, a pesquisa foi caracterizada como estudo de caso, de caráter exploratório, com uma abordagem qualitativa, fazendo uso de entrevista para obter os dados necessários à pesquisa. A análise dos dados foi realizada através da transcrição da fala do entrevistado. Os resultados apontam que o setor alimentício do hospital oferece condições adequadas para a elaboração dos pratos ofertados, pois o setor preocupa-se com a qualidade, armazenamento e procedência dos alimentos, visando o bem-estar daqueles que os consomem. Este trabalho conclui que além de ofertar hospitalidade ao tratamento do usuário do serviço de saúde, o setor de alimentos e bebidas tem um papel importante na recuperação e tratamento do cliente.
\end{abstract}

Palavras-chaves: Alimentos e Bebidas. Hotelaria Hospitalar. Hospitalidade.

\section{HOSPITAL HOSPITALITY: A CASE STUDY ON THE FOOD AND BEVERAGE SERVICE AT THE MUNICIPAL HOSPITAL OF JAGUARIBE-CEARÁ}

\begin{abstract}
This research addresses the food and beverage sector within the hospitality industry. The general objective of the study was to analyze the organization of the food and beverage sector of a public hospital in Ceará. The specific objectives were: to ascertain what the main foods offered by the institution and its relation with the rehabilitation and hospitality; identify how the elaboration of the menu and the professionals involved in this process occur; verify the origin and storage of food. As for the methodology, the research was characterized as an exploratory case study, with a qualitative approach, making use of interview to obtain the necessary data for the research. Data analysis was performed through the transcript of the interviewee's speech. The results indicate that the food sector of the hospital offers adequate conditions for the elaboration of the dishes offered, since the sector is concerned with the quality, storage and origin of the food, aiming the well-being of those who consume them. This paper concludes that in addition to offering hospitality to the treatment of the health service user, the food and beverage sector plays an important role in the recovery and treatment of the client.
\end{abstract}

Keywords: Food and Beverage. Hospital Hospitality. Hospitality.

\section{INTRODUÇÃO}

A hotelaria busca alcançar a satisfação dos clientes, inclusive daqueles que se encontram em um leito hos- 
pitalar. Os usuários de um serviço de saúde devem ser tratados com cortesia, agilidade, cordialidade, ou seja, hospitalidade. Estes tem o direito de segurança, conforto e bem-estar, garantindo assim, a qualidade do atendimento.

O conceito de hotelaria hospitalar é algo novo devido à indispensabilidade dos hospitais em ofertar além de cura ou tratamento, resguardo, consolação e principalmente aconchego aos seus clientes, familiares e visitantes, transformando o ambiente hospitalar em algo mais acolhedor e confortável (BRITO; PERINOTTO 2014).

A hotelaria hospitalar busca satisfazer as necessidades dos usuários de um serviço de saúde, na recepção, no setor de governança e no setor de alimentos e bebidas, sendo este último, responsável por ofertar uma dieta rica em nutrientes, a fim de contribuir para a melhoria do paciente. Em instituições hospitalares, o setor de alimentos e bebidas deve elaborar cardápios que mantenham as peculiaridades de sabor e odor e as refeições devem ser atrativas e apetitosas (MASCARENHAS; MAINARDES; CARNEIRO, 2016).

Os funcionários do setor alimentício de um hospital, devem garantir a produção de refeições com qualidade, partindo do ponto de vista nutricional, garantindo a segurança alimentar em suas preparações. Sobretudo, para assegurar a qualidade da alimentação, deve-se fornecer aos colaboradores, habilidades necessárias para torná-los aptos a desenvolverem suas atividades na área de alimentos (KULMANN; GIACOMELLI, 2015).

A relevância da pesquisa consiste em apresentar o setor de alimentos e bebidas nos aspectos referentes à hospitalidade do usuário de um serviço de saúde, tendo a dieta como um recurso que visa auxiliar na recuperação do indivíduo no ambiente hospitalar. Os conhecimentos do setor de alimentos e bebidas para profissionais e estudantes da área de gastronomia, turismo e saúde, assim como para os usuários dos serviços de saúde e para as instituições públicas e particulares deste âmbito, discutidos aqui, são essenciais para a formação e prática laboral dos mesmos.

Foi elaborada a seguinte pergunta de partida, de cunho investigativo para este estudo: como se caracteriza a organização do setor de alimentos e bebidas de um serviço de saúde para melhor atender o usuário do serviço?

O objetivo geral desta pesquisa é analisar a organização do setor de alimentos e bebidas de um serviço de saúde. Os objetivos específicos foram: averiguar quais os principais alimentos oferecidos pela instituição e sua relação com a recuperação e hospitalidade; identificar como ocorre a elaboração do cardápio e os profissio- nais envolvidos neste processo; verificar a procedência e armazenamento dos alimentos.

Quanto à metodologia, trata-se de um estudo de caso, de caráter exploratório, com uma abordagem qualitativa, envolvendo um hospital municipal do Estado do Ceará. A coleta de dados foi realizada através da aplicação de uma entrevista semiestruturada com o responsável pelo setor de alimentos e bebidas da instituição, em questão. A análise dos dados foi realizada através da transcrição das falas do entrevistado.

Este estudo apresenta seções sobre conceitos e definições de hotelaria hospitalar, assim como o setor de alimentos e bebidas no referido ambiente; a metodologia para a obtenção dos dados; a discussão dos resultados obtidos; e as considerações sobre a pesquisa.

\section{FUNDAMENTAÇÃO}

\subsection{Hotelaria Hospitalar}

A hotelaria hospitalar pode ser entendida como um aglomerado de serviços de hotelaria clássica, adequado a um serviço de saúde, com o intuito de proporcionar bem-estar ao cliente. Segundo Boeger (2003), a hotelaria hospitalar é um agrupamento de funções de apoio, em conjunto com serviços peculiares, que proporcionam aos clientes (internos e externos) de saúde, segurança no período de internação e conforto.

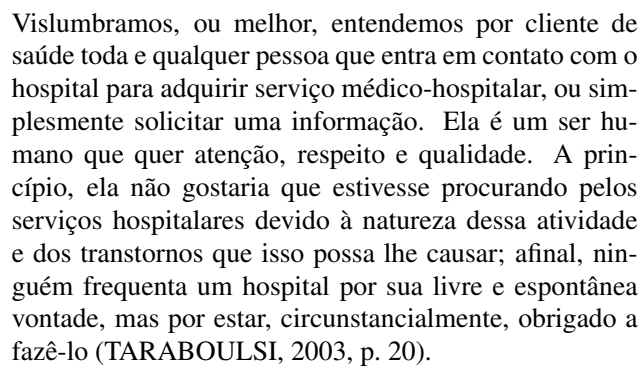
saúde toda e qualquer pessoa que entra em contato com o hospital para adquirir serviço médico-hospitalar, ou simplesmente solicitar uma informação. Ela é um ser humano que quer atenção, respeito e qualidade. A princípio, ela não gostaria que estivesse procurando pelos serviços hospitalares devido à natureza dessa atividade e dos transtornos que isso possa lhe causar; afinal, ninguém frequenta um hospital por sua livre e espontânea vontade, mas por estar, circunstancialmente, obrigado a fazê-lo (TARABOULSI 2003. p. 20).

A hotelaria hospitalar envolve os serviços oferecidos em uma instituição de saúde muito similares ao de um meio de hospedagem tradicional, sobretudo, reconhece que se trata de uma clientela mais vulnerável e com exigências diferenciadas. Portanto, melhora-se a infraestrutura do ambiente hospitalar, para ofertar mais qualidade ao serviço (BRITO; PERINOTTO, 2014).

\footnotetext{
O triunfo da hotelaria está na humanização do ambiente hospitalar: serviços eficientes que encantam, cores suaves, plantas e jardins bem cuidados e, principalmente, pessoas entusiasmadas interagindo com os clientes de saúde, revelando de tal forma o segredo dessa nova proposta que é o sorriso sincero e permanente, marca registrada da satisfação e do amor pelo trabalho realizado. Os clientes de saúde (enfermos, familiares, acompanhantes, visitantes) sentem-se confiantes e motivados
} 
quando a solidariedade se apresenta estampada nos semblantes de todos os envolvidos no seu atendimento TARABOULSI 2003. p. 40).

Os clientes não só procuram os benefícios dos serviços de saúde, mas também, buscam respeito e solidariedade, pois como estão em uma situação vulnerável, necessitam de um ambiente adequado que atenda o seu estado físico, assim como qualidade no atendimento para o seu estado emocional (TARABOULSI, 2004).

Para Godoi (2008) a hotelaria hospitalar trouxe consigo diversos benefícios como a melhoria dos atendimentos humanitários, nas alas pediátricas com espaços voltados exclusivamente para crianças internadas. Para atender às novas demandas do âmbito hospitalar, a gestão deve incorporar novas ações e acompanhar as tendências da área, principalmente as de hospitais modernos.

De acordo com Ruthes e Cunha (2007), o hospital moderno é aquele que passou por um processo de transformação em sua forma de gestão, incorporando uma visão de negócios. Além disso, trata-se de um tipo de empresa que implica grande divisão de tarefas, coexistindo habilidades e técnicas variadas.

Os novos hospitais, enquadrados no contexto de hotelaria hospitalar possuem características como: portas automáticas na entrada, recepção informatizada e padronizada, sala de espera aconchegante, com cadeiras confortáveis, televisão e climatização, elevadores sofisticados dentre outros.

Os quartos possuem decoração diferenciada, com todas características de um meio de hospedagem clássico, como: telefone, televisão, ar condicionado, dentre outros. Alguns hospitais terceirizam o serviço de alimentação, outros administram este setor, que é responsável pelas refeições oferecidas aos pacientes e acompanhantes.

\begin{abstract}
As paredes podem ser pintadas em tom de pastel e o banheiro pode ser revestido com tinta à base de água, lavável, evitando-se, dessa forma, o uso de azulejos e, assim, as famosas emendas entre as lajotas, que podem ser foco de sujeira e proliferação de bactérias, o que resulta, em proporções maiores, até em infecção hospitalar. Ainda no banheiro, deve-se evitar a instalação de boxe de vidro ou de plástico, por exigir emendas, trilhos, maçanetas e dobradiças, que dificultam a higienização. Todas essas peças com formatos de difícil acesso à limpeza podem tornar-se foco de proliferação de sujeira e incrementar a infecção hospitalar. Além disso, os trilhos dos boxes de plástico criam um obstáculo para o acesso da cadeira de banho a pacientes dependentes desse equipamento (BOEGER 2003 p. 31).
\end{abstract}

Há uma diferença entre as gestões dos hospitais privados e públicos. Porém, Boeger (2003, p. 22), afirma que: "apesar de muitos hospitais públicos enfrentarem dificuldades adicionais se comparados com hospitais privados, há algum deles que conseguem, com criatividade e competência, desenvolver trabalhos tão bons quanto os de alguns hospitais privados".

Esta tendência nos ambientes hospitalares surgiu para efetivar a hospitalidade. Castelli (2005) considera a hospitalidade como o ato de bem receber o indivíduo, com atenção durante a permanência da pessoa no espaço físico, até o momento da despedida. O hóspede ou paciente precisa ter suas expectativas atendidas para considerar a qualidade na hospitalidade.

Já Dencker e Bueno (2003, p. 19) afirmam que "hospitalidade, do ponto de vista analítico operacional, pode ser definida como o ato humano, exercido em contexto doméstico, público ou profissional, de recepcionar, hospedar, alimentar e entreter pessoas temporariamente deslocadas de seu habitat".

Estudos apontam que a melhora do ambiente hospitalar atrelada aos serviços humanizados, trazem benefícios como a minimização do tempo de internação, elevação do bem-estar geral dos pacientes e funcionários, redução das faltas de trabalho na equipe de saúde, e consequente redução de gastos hospitalares (MARTINS, 2001; MAZZETTI, 2005). Logo, trata-se de benefícios mútuos para os empreendimentos hospitalares, funcionários e clientes de saúde.

\subsection{Alimentos e Bebidas no Contexto Hospitalar}

A gastronomia envolve a arte do preparo, conhecimentos e técnicas para a cocção e escolha adequada dos ingredientes utilizados na produção de determinado prato, afinal, o mesmo deve atender às expectativas de quem ingere (Brillat Savarin, 1995).

\footnotetext{
Os alimentos e bebidas desempenham um papel importante na definição da identidade de grupos, comunidades e sociedades, bem como na definição do relacionamento entre os indivíduos e o contexto social mais amplo. Somos o que comemos (LASHLEY; MORRISON 2004. p. 12).
}

Na unidade hospitalar, o setor de alimentação e nutrição é responsável pela produção de refeições. O setor tem a responsabilidade de comprar, receber, guardar e preparar os alimentos para finalmente serem consumidos (NONINO-BORGES et al., 2006).

De acordo com Lody (2007) a qualidade final de um produto alimentício está relacionada com todas as etapas da cadeia de suprimentos, desde as ações do fornecedor dos produtos, ou seja, a entrega deve estar enquadrada dentro dos padrões regidos pelo Ministério da Saúde até a adequada armazenagem dos produtos.

Nakasato e Souza (2011), afirmam que a gastronomia hospitalar é a arte de conciliar a prescrição dieté- 
tica e o controle alimentar de clientes à composição de refeições saudáveis, nutritivas, atrativas e saborosas, a fim de gerar a associação de objetos dietéticos, clínicos e sensoriais e promovendo uma nutrição adequada. A alimentação e a nutrição são conjunções básicas para a melhoria e a proteção da saúde (BADARÓ; AZEREDO; ALMEIDA, 2007).

A gastronomia hospitalar tem como principal objetivo manter e/ou recuperar o estado nutricional do paciente internado, e deve reduzir o sofrimento deste indivíduo que está separado de suas atividades familiares, sociais, trabalhistas e outras (MESSIAS; PRESTA; SOUZA, 2011). Nos hospitais, a qualidade dos alimentos é um dos itens que mais tem influência no nível de satisfação do paciente internado (TONTINI; WALTER; COSTA, 2012).

\begin{abstract}
O serviço de gastronomia, conhecido pelo modelo de gestão tradicional por Serviço de Nutrição e Dietética (SND) dentro dos hospitais pode ser considerado outra tendência e exigência deste "novo" consumidor de saúde. O modelo de gestão do hospital moderno deve compreender a importância desta área e a grande oportunidade de encantamento do cliente através da gastronomia, já que esta é uma área com o antigo paradigma de comida de hospital ser associada à alimentação de baixa qualidade e nenhum atrativo (BOEGER 2006 p. 40).
\end{abstract}

As dietas são compostas considerando-se o estado nutricional e fisiológico dos indivíduos, e em situações hospitalares, deve adequar-se à situação clínica dos clientes, além de proporcionar benfeitoria à recuperação dos mesmos (ISOSAKI; CARDOSO; OLIVEIRA 2009).

Segundo Boeger (2006, p. 40) "além dos aspectos gastronômicos e estéticos inerentes à Gastronomia Hospitalar, a dieta do paciente pode ajudá-lo na sua melhor recuperação". Observa-se que a área de alimentos e bebidas está inserida nos hospitais como um diferencial, uma forma de humanização no serviço oferecido. As dietas passam a ser imprescindíveis ao cliente de saúde.

\section{METODOLOGIA}

Tratou-se de um estudo de caso, de caráter exploratório, com uma abordagem qualitativa. O cenário da pesquisa foi um hospital municipal do Estado do Ceará, integrante do Sistema Único de Saúde (SUS). Quanto aos meios de investigação, a primeira etapa da pesquisa foi caracterizada por uma pesquisa bibliográfica. A pesquisa bibliográfica tem como finalidade elucidar e amplificar o conhecimento sobre um evento específico (MARCONI; LAKATOS, 2006).

A segunda etapa da pesquisa foi de caráter exploratório, proporcionando maior familiaridade com o pro- blema. Para tal, utilizou-se uma entrevista semiestruturada com o profissional responsável pelo setor de alimentos e bebidas do hospital, em questão. A entrevista foi aplicada em maio de 2016 e possuía oito perguntas. A entrevista foi gravada e a análise dos dados foi realizada através da transcrição das falas do entrevistado, que foi identificado neste estudo através da letra X.

\section{RESUltados E DISCUSSÕES}

Nesta seção apresentam-se os resultados coletados através de uma entrevista semiestruturada e suas respectivas discussões. A pesquisa foi realizada com o responsável pelo setor de alimentos e bebidas do hospital municipal cearense, participante desta pesquisa. $\mathrm{O}$ entrevistado respondeu a oito perguntas.

A primeira pergunta da entrevista versou sobre a organização do setor de alimentos e bebidas do hospital, como resultado obteve-se que os alimentos são organizados em um ambiente próprio e há o controle dos mesmos por prazo de validade, como pode ser observado na fala a seguir.

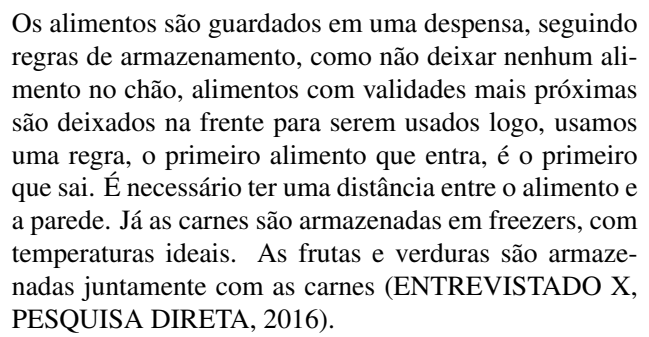

Os alimentos são armazenados de forma correta no hospital, a equipe deste setor está atenta às normas de segurança e ao controle de validade dos produtos. De acordo com a Agência Nacional de Vigilância Sanitária (ANVISA) os alimentos e/ou produtos perecíveis: alimentos in natura, alimentos preparados e prontos para o consumo precisam de condições de temperatura ideal para a sua conservação (ANVISA, 2000). Observase que o hospital atende às normas de vigilância, ao preocupar-se com as temperaturas ideais de armazenamento.

O segundo questionamento abordou a procedência dos alimentos que chegam ao hospital. Os resultados apontam que os alimentos são comprados de fornecedores confiáveis e passam por inspeção no momento da entrega, como pode ser verificado na fala a seguir.

\footnotetext{
Os alimentos são comprados de fornecedores particulares, de acordo com as licitações realizadas anualmente. Todos os alimentos passam primeiro por um processo de fiscalização ao serem entregues, depois os cuidados tomados são no armazenamento, higienização local, validade dos alimentos, estado das embalagens, até a higie-
} 
nização na hora da preparação dos alimentos (ENTREVISTADO X, PESQUISA DIRETA, 2016).

Nota-se uma preocupação com a procedência dos alimentos, além disso, há um protocolo realizado para que o alimento recebido tenha qualidade no momento do preparo e isto é de suma importância, pois em um ambiente de tratamento e recuperação, como é o hospital, os serviços de alimentos e bebidas devem evitar contaminações para não causar mais danos à saúde dos usuários do serviço.

A terceira questão buscou identificar como os alimentos produzidos no hospital podem ajudar ao paciente internado, o resultado obtido foi satisfatório, pois a alimentação no ambiente hospitalar foi apontada como uma forma de nutrir um organismo debilitado em recuperação e/ou tratamento, logo, observa-se a preocupação com a nutrição e a dietética.

\section{A alimentação é rica de nutrientes necessários ao corpo humano, quando a dieta é feita envolvendo o máximo de nutrientes possíveis, o organismo terá suas necessidades atendidas, por isso, o cuidado com o cardápio do hospi- tal, pois a alimentação é a força que o organismo precisa para reagir ao tratamento ou recuperação no ambiente hospitalar (ENTREVISTADO X, PESQUISA DIRETA, 2016).}

Para Sousa et al. (2013), a condição nutricional do paciente hospitalizado está diretamente relacionada à conduta nutricional implementada, ou seja, a alimentação adequada para atender às carências nutricionais durante a hospitalização, depende de como o setor de alimentos e bebidas irá organizar seu cardápio, considerando as individualidades de cada indivíduo.

De acordo com Sousa (2002), o ato de comer não é apenas um componente de conforto para o paciente no período de internação hospitalar, mas uma necessidade vital. Para que haja a recuperação total dos pacientes hospitalizados é vital que os mesmos sejam bem amparados tanto no que diz respeito ao tratamento medicamentoso, como nutricional.

A quarta questão referiu-se à existência de algum responsável por fiscalizar a chegada dos alimentados à área hospitalar, como resultado obteve-se que existe um profissional responsável para esta ação, como pode ser observado na fala abaixo.

A primeira fiscalização é feita por uma pessoa responsável por conferir os pesos e se faltou algum produto solicitado. Após esta conferência, os alimentos são conferidos mais uma vez, agora pela nutricionista, que fiscaliza a qualidade e validade dos alimentos, para evitar que o hospital receba algum alimento fora dos padrões de qualidade (ENTREVISTADO X, PESQUISA DIRETA, 2016).
Considerando a relevância de fiscalizar as particularidades dos alimentos, é vital que a instituição tenha alguém responsável para esta tarefa. Com todos esses cuidados, é possível manter a qualidade dos alimentos nas dependências do hospital, uma vez que os mesmos passam pela triagem de dois profissionais, minimizando possíveis erros no recebimento.

A quinta questão buscou saber quais as necessidades dos pacientes hospitalizados, como resultado obteve-se que as dietas são individualizadas, como pode ser observado a seguir: " as necessidades variam de acordo com a patologia do usuário do serviço, sendo assim, a dieta pode ser: hipossódica, para diabético, pastosa, branda, dieta zero" (ENTREVISTADO X, PESQUISA DIRETA, 2016).

É importante que cada paciente seja tratado de forma individualizada, pois cada cliente tem necessidades especiais, como por exemplo, pacientes diabéticos e hipertensos, que requerem atenção especial quanto à quantidade de açúcares e sal nas refeições, respectivamente.

A sexta questão buscou identificar como é realizado o trabalho da equipe de nutrição, como a equipe está dividida, para melhor compreensão dos processos de trabalho do setor. Os resultados apontam que os o trabalho acontece de forma integrada para a obtenção de melhores resultados, como pode ser observado na fala, a seguir.

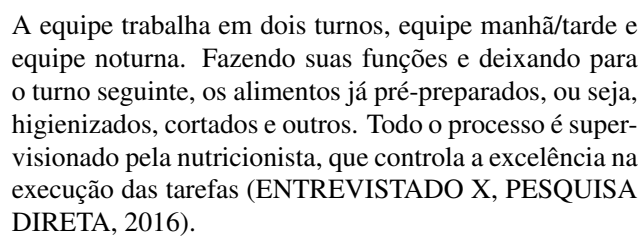

A equipe trabalha em dois turnos, equipe manhã/tarde e equipe noturna. Fazendo suas funções e deixando para o turno seguinte, os alimentos já pré-preparados, ou seja, higienizados, cortados e outros. Todo o processo é supervisionado pela nutricionista, que controla a excelência na execução das tarefas (ENTREVISTADO X, PESQUISA DIRETA, 2016).

Observa-se que a nutricionista está presente em todas as ações do setor de alimentos e bebidas na unidade hospitalar, o que é algo positivo, pois de fato corrobora com o controle, e por conseguinte, qualidade dos serviços do setor. As competências do nutricionista nos hospitais são situadas em duas grandes áreas de atuação: a alimentação coletiva, relacionadas ao gerenciamento do processo de manipulação de refeições e a nutrição clínica que compreende atividades relacionadas às necessidades nutricionais dos pacientes hospitalizados (SOUSA, 2002).

A sétima questão versou sobre alimentação humanizada, como resultado obteve-se que se trata de um atendimento individualizado, suprindo as necessidades do paciente, independente de raça, gênero, classe social e outros, como pode ser observado na fala abaixo.

A alimentação humanizada é o atendimento individualizado para todos os internados, independente de raça, cor, 
condição social, focando no lado humano. A elaboração dos pratos, apresentação dos mesmos, talheres nas bandejas, variação dos cardápios, cada detalhe, não esquecendo as condições higiênicas e sanitárias. Com isso aumentamos a satisfação dos pacientes, e consequentemente a aceitação das dietas hospitalares (ENTREVISTADA X, PESQUISA DIRETA, 2016).

É de extrema importância que todos os indivíduos sejam tratados com respeito e recebam a melhor forma de atendimento. Além disso, todo ser humano tem o direito de ser bem assistido em qualquer ambiente, inclusive o hospitalar.

Por fim, a oitava e última questão, buscou analisar o processo de avaliação do cardápio hospitalar e com resultado, obteve-se que o cardápio é planejamento para o mês, como pode ser observado na fala abaixo.

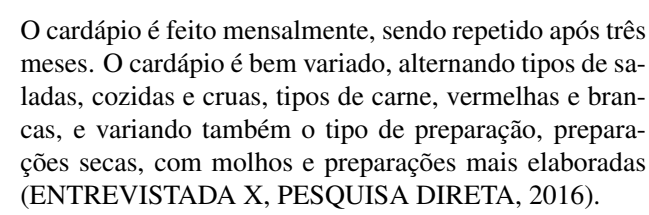

O cardápio é feito de forma diversificada, buscando satisfazer os clientes, considerando as restrições do usuário. Ter opções no cardápio é fundamental, pois às vezes um usuário para um longo período hospitalizado, então se não houver variação na alimentação, o mesmo passará a recusar o que lhe é ofertado.

\section{CONSIDERAÇÕES FINAIS}

A pesquisa apresentou a hotelaria hospitalar, envolvendo os processos de trabalho do setor alimentício de um hospital municipal cearense, no intuito de detalhar suas diretrizes, bem como o funcionamento da gastronomia hospitalar para garantir a hospitalidade do usuário do serviço de saúde.

O objetivo geral deste estudo foi atingido, pois o setor alimentício do hospital municipal, em questão, oferece condições adequadas para a excelência no armazenamento e elaboração dos pratos ofertados, pois o setor preocupa-se com a compra de alimentos de boa qualidade e procedência segura, visando o bem-estar daqueles que o consomem.

O objetivo específico referente aos principais alimentos oferecidos pela instituição e sua relação com a reabilitação e hospitalidade, obteve êxito, pois foi observado que os principais alimentos são frutas, verduras, legumes, carnes, existe a oferta de um cardápio amplo, garantindo aos pacientes maiores condições para sua recuperação.

Quanto à elaboração do cardápio e os profissionais envolvidos neste processo, o objetivo foi atingido, pois a elaboração do cardápio tem a finalidade de contemplar as necessidades individuais dos pacientes quanto às restrições de cada um, no intuito de atender da melhor forma possível as carências e peculiaridades dos clientes de saúde, esse processo é da responsabilidade da equipe de nutrição da instituição.

A verificação da origem e armazenamento dos alimentos obteve êxito, pois os alimentos são de boa procedência, garantido assim a segurança necessária para o consumo, os mesmos são estocados em ambiente propício e organizados por prazo de validade, a fim de garantir o controle do estoque.

Este estudo aponta possibilidades de pesquisas posteriores como: uma análise da equipe multidisciplinar envolvida no setor de alimentação; a qualidade dos alimentos preparados no setor de alimentos e bebidas dos serviços de saúde na perspectiva do acompanhante; a qualidade dos alimentos ofertados na perspectiva do cliente.

\section{REFERÊNCIAS}

ANVISA, A. N. D. V. S. Resolução no 23, de 15 de março de 2000. 2000. Disponível em: $<$ http://portal.anvisa.gov.br/wps/wcm/connect/ 2b82600040b2ac328be3dbf7d85acaec/Microsoft+ Word+-+RESOLU\%C3\%87\%C3\%83O+N\%C2\%BA+ 23,+DE+15+DE+MAR\%C3\%87O+DE+2000.pdf? MOD=AJPERES $>$. Acesso em: 30 jun. 2016.

BADARÓ, A.; AZEREDO, R.; ALMEIDA, M. d. Vigilância sanitária de alimentos: uma revisão. Revista Digital de Nutrição: Nutrir Gerais, Unileste-MG, v. 1, n. 1, p. 1-25, 2007.

BOEGER, M. A. Gestão em Hotelaria Hospitalar. São Paulo, 2003.

Hotelaria hospitalar como modelo de gestão nas Instituições de saúde particulares de grande porte no município de São Paulo. São Paulo, 2006.

Brillat Savarin, J. A. A fisiologia do gosto. São Paulo, 1995.

BRITO, L. S.; PERINOTTO, A. R. C. O brincar como promoção à saúde: a importância da brinquedoteca hospitalar no processo de recuperação de crianças hospitalizadas. Revista Hospitalidade, v. 1, n. 1, p. 291-315, 2014.

CASTELLI, G. Hospitalidade: na perspectiva da Gastronomia e da Hotelaria. São Paulo, 2005.

DENCKER, A. F. M.; BUENO, M. S. Hospitalidade: cenários e oportunidades. São Paulo, 2003. 
GODOI, A. F. Hotelaria hospitalar e humanização no atendimento em hospitais. 2. ed. São Paulo: Ícone, 2008 .

ISOSAKI, M.; CARDOSO, E.; OLIVEIRA, A. D. Manual de dietoterapia e avaliação nutricional: Serviço de Nutrição e dietética do Instituto do Coração-HCFMUSP. 2. ed. São Paulo: Atheneu, 2009.

KULMANN, I. S.; GIACOMELLI, S. C. Gastronomia hospitalar: proposta de técnicas gastronômicas por meio de capacitação. In: Anais do VII Salão Internacional de Ensino, Pesquisa e Extensão. [S.1.]: Universidade Federal do Pampa, 2015.

LASHLEY, C.; MORRISON, A. Em busca da hospitalidade: perspectivas para um mundo globalizado. Barueri, 2004.

LODY, R. Comer até o talo ou patrimonialização da comida. In: Saber e Sabor I Colóquio de História e Cultura da Alimentação. [S.1.: s.n.], 2007.

MARCONI, M. A.; LAKATOS, E. M. Fundamentos de metodologia científica. 5. ed. São Paulo: Atlas, 2006.

MARTINS, M. C. F. Humanização das relações assistenciais de saúde: a formação do profissional de saúde. São Paulo, 2001.

MASCARENHAS, R. G. T.; MAINARDES, I.; CARNEIRO, D. C. Eventos em hotelaria hospitalar: Aspectos da gastronomia no fornecimento de cardápio para dietas restritivas/events in hospital hotels: Aspects of gastronomy in menu supply for restrictive diets. ROSA DOS VENTOS-Turismo e Hospitalidade, v. 8, n. 1, p. 1-12, 2016.

MAZZETTI, M. Especialistas garantem benefícios de humanização de hospitais. 2005. Disponível em: <http://www1.folha.uol.com.br/folha/equilibrio/ noticias/ult263u3854.shtml> Acesso em: 27 mai. 2016.

MESSIAS, G.; PRESTA, F.; SOUZA, M. Benefícios da gastronomia hospitalar na alimentação do paciente idoso. Revista Eletrônica Novo Enfoque, v. 12, n. 12, p. 23-31, 2011.

NAKASATO, M.; SOUZA, M. D. A gastronomia hospitalar auxiliando na redução dos índices de desnutrição entre pacientes hospitalizados. [S.l.], 2011.
NONINO-BORGES, C. B.; RABITO, E. I.; SILVA, K. da; FERRAZ, C. A.; CHIARELLO, P. G.; SANTOS, J. S. dos; MARCHINI, J. S. Desperdício de alimentos intra-hospitalar food wastage in a hospital. Revista de Nutrição, Directory of Open Access Journals, v. 19, n. 3, p. 349-356, 2006.

RUTHES, R. M.; CUNHA, I. Os desafios da administração hospitalar na atualidade. Revista de Administração em Saúde, v. 9, n. 35, p. 93-102, 2007.

SOUSA, A. A interação entre a terapia nutricional e a produção de refeições: repensando a função da alimentação hospitalar. Nutrição em pauta, v. 53, n. 1, p. 17-21, 2002.

SOUSA, A. A.; SALLES, R. K. de; ZILIOTTO, L. F.; PRUDENCIO, A. P. A.; MARTINS, C. A.; PEDROSO, C. G. T. Hospital food: elements to build humanizing initiatives/alimentacao hospitalar: elementos para a construcao de iniciativas humanizadoras. Demetra: Food, Nutrition \& Health, Universidade do Estado do Rio de Janeiro-Uerj, v. 8, n. 2, p. 149-163, 2013.

TARABOULSI, F. A. Administração de Hotelaria Hospitalar. São Paulo, 2003.

Administração de hotelaria hospitalar: serviços aos clientes, humanização do atendimento, departamentalização, gerenciamento, saúde e turismo, hospitalidade. São Paulo, 2004.

TONTINI, G.; WALTER, S. A.; COSTA, M. Satisfação com serviços hospitalares como fator estratégico de gestão: uma abordagem não linear. In: ANAIS (Ed.). XV Simpósio de Administração da Produção, Logística e Operações Internacionais. São Paulo, 2012. 